\title{
Otitis media aguda. Diagnóstico y tratamiento
}

\author{
M. Elena Santolaya de $P$.
}

\section{Acute otitis media. Diagnosis and treatment}

Acute otitis media (AOM) is one of the most common causes of medical visit and antimicrobial use in children. A rationale management approach requires a thorough clinical exam and updated knowledge on local patterns of microorganisms involved and antimicrobial susceptibility profiles. Clinical diagnosis should be performed with pneumatic otoscopy. The most common microorganism causing AOM in Santiago, Chile according to local studies are Streptococcus pneumoniae (40\%), non-capsulated Haemophilus influenzae (29\%), Streptococcus pyogenes (7\%) and Moraxella catarrhalis (4\%). S. pneumoniae has acquired resistance to penicillin in the last decade, resistance that has been extrapolated to other $\beta$-lactams such as amoxicillin, reason why broader spectrum antimicrobials are routinely prescribed. Clinical practice has consistently shown although that the great majority of children receiving amoxicillin at a dose of $80-100 \mathrm{mg} / \mathrm{kg} / \mathrm{day}$ resolve their AOM. Recent studies from our group have demonstrated that resistance to penicillin can not be extrapolated to amoxicillin. In vitro high level resistance to penicillin vs amoxicillin is $18 \%$ vs $0.5 \%$. Based on this data, our current recommendation for AOM is amoxicillin $80 \mathrm{mg} / \mathrm{kg} / \mathrm{day}, \mathrm{q} 12$ hours for 10 days in infants and for 5-7 days in children $>2$ years of age who have not had an episode within the previous month. For amoxicillin failures, amoxicillin $+\beta$ lactam inhibitor or a second generation cephalosporin are recommended, especially in areas with a high prevalence of $\beta$ lactam producing $H$. influenzae and $M$. catarrhalis. Treatment of children with AOM universally require appropriate follow-up in order to comply with the proposed algorithm.

Key words: Acute otitis media, etiology, treatment.

Palabras claves: Otitis media aguda, etiología, tratamiento.

\section{Definición}

$\mathbf{L}$ a otitis media aguda (OMA) se define como la presencia de efusión timpánica, demostrada por neumo-otoscopia, nivel hidroaéreo o impedanciometría, acompañada de signos y síntomas de inflamación aguda del oído medio. Los síntomas más característicos son otalgia, fiebre e irritabilidad; y los signos clásicos son otorrea y/o alguna de las siguientes alteraciones de la membrana timpánica: inflamación, engrosamiento y/o abombamiento, opacidad, presencia de bulas, depósito de fibrina, coloración blanco amarillenta y ausencia de movimiento a la neumootoscopia.

\section{Diagnóstico}

Las infecciones respiratorias constituyen la principal causa de consulta en pediatría y, dentro de éstas, OMA representa la principal indicación de uso de antimicrobianos. El manejo racional de esta patología implica un diagnóstico acucioso y un conocimiento actualizado de las especies bacterianas involucradas en su etiología y de la susceptibilidad in vitro a distintos antimicrobianos.

El diagnóstico certero de una OMA parece teóricamente sencillo de acuerdo con la definición ya expuesta pero, en la práctica, existe un importante sobrediagnóstico. En una experiencia nacional, en 529 niños derivados desde un servicio de urgencia al otorrinolaringólogo con diagnóstico de OMA, 44\% tuvo una OMA confirmada por neumo-otoscopia y punción timpánica, y el $56 \%$ restante, tuvo los siguientes diagnósticos: otoscopia normal (34\%), otitis media aguda con efusión $(14 \%)$, otitis media crónica $(5 \%)$ y otitis externa (3\%). Es relevante destacar en este punto, que el pediatra general se ve expuesto a examinar, continuamente, lactantes febriles con un cuadro respiratorio agudo de etiología viral, que lloran al momento del examen físico, en que la membrana timpánica aparece como inflamada o abombada. El mejor consejo para un diagnóstico más acucioso, es realizar una neumootoscopia, en que la presencia de movimiento de la membrana timpánica, descarta la presencia de líquido en el oído medio y, por lo tanto, hace muy improbable el diagnóstico de OMA.

\author{
Universidad de Chile, \\ Santiago, Chile \\ Facultad de Medicina \\ Departamento de Pediatría Oriente \\ Hospital Luis Calvo Mackenna, \\ Santiago, Chile \\ Unidad de Infectología \\ Recibido: 18 agosto 2006 \\ Aceptado: 16 octubre 2006 \\ Correspondencia a: \\ María Elena Santolaya de Pablo \\ msantola@med.uchile.cl
}




\section{Etiología, susceptibilidad y mecanismos de resistencia a antimicrobianos}

En estudios realizados en 543 niños con OMA de los sectores Sur Oriente y Oriente de Santiago, se ha visto que el principal agente involucrado en OMA es Streptococcus pneumoniae (40\%), seguido de Haemophilus influenzae no capsulado (29\%), Streptococcus pyogenes $(7 \%)$ y Moraxella catarrhalis (4\%) (Tabla 1).

En la última década, S. pneumoniae ha desarrollado resistencia a penicilina (PNC), principalmente, a través de mutaciones de proteínas de unión que se encuentran en la pared de la bacteria. Se definen tres rangos de susceptibilidad a PNC: Susceptible: $\mathrm{CIM} \leq 0,06 \mu \mathrm{g} / \mathrm{ml}$, resistencia intermedia: CIM $>0,06 \mathrm{y} \leq 1 \mu \mathrm{g} / \mathrm{ml}$, y alta resistencia: CIM $>1 \mu \mathrm{g} / \mathrm{ml}$. La resistencia de $S$. pneumoniae a PNC en 187 cepas aisladas de fluido de oído medio en niños con OMA en Santiago de Chile, fue de 18\% (Tabla 2).

Tabla 1. Especies bacterianas aisladas de fluido de ó́do medio en 543 niños con otitis media aguda

\begin{tabular}{lrl} 
Patógeno & (n = 543) \\
S. pneumoniae & 216 & $(40)$ * \\
H. influenzae & 156 & $(29)$ \\
\hline B-lactamasa positivo & $16 / 156$ & $(10)$ \\
S. pyogenes & 37 & $(7)$ \\
M. catarrhalis** & 23 & $(4)$ \\
Otros*** & 37 & $(7)$ \\
Negativo & 74 & $(13)$ \\
& & \\
* Números en paréntesis, porcentaje del total & \\
** 100\% M. catarrhalis fueron $\beta$-lactamasa positiva \\
*** Otros incluye Staphylococcus aureus, Staphylococcus \\
coagulasa negativa, Pseudomonas sp, Streptococcus grupo \\
viridans, Neisseria sp, Proteus mirabilis
\end{tabular}

\begin{tabular}{|c|c|c|c|}
\hline \multicolumn{4}{|c|}{$\begin{array}{l}\mathbf{N}^{\circ} \text { de cepas susceptibles de resistencia intermedia y } \\
\text { resistentes a PNC* y AMX* }\end{array}$} \\
\hline Susceptibilidad & $\begin{array}{c}\text { PNC } \\
n=187\end{array}$ & $\begin{array}{l}\text { AMX } \\
n=187\end{array}$ & Valor de $\mathbf{P}$ \\
\hline Susceptible & $112(60)^{* *}$ & $177(94,7)$ & $<0,05$ \\
\hline Resistencia intermedia & $41(22)$ & $9 \quad(4,8)$ & $<0,05$ \\
\hline Resistencia & $34(18)$ & $1 \quad(0,5)$ & $<0,05$ \\
\hline \multicolumn{4}{|c|}{$\begin{array}{l}\text { * PNC: penicilina; AMX: amoxicilina. Susceptibidad determinada por E test } \\
\text { ** Número en paréntesis, porcentaje del total }\end{array}$} \\
\hline
\end{tabular}

Haemophilus influenzae y M. catarrhalis tienen como principal mecanismo de resistencia la producción de $\beta$ lactamasas, lo que les confiere resistencia a antimicrobianos $\beta$ lactámicos de primera línea. En el caso de $H$. influenzae, $10 \%$ de 156 cepas aisladas de niños con OMA en nuestro medio, fueron productoras de $\beta$ lactamasa, lo que en $M$. catarrhalis ascendió a $100 \%$. Streptococcus pyogenes es una bacteria que, con el paso del tiempo, mantiene intacta su susceptibilidad a PNC y otros $\beta$ lactámicos, no reportándose casos de resistencia en Chile ni en el resto del mundo. Se ha reportado, sin embargo, resistencia de $S$. pyogenes a macrólidos, a través de un mecanismo de eflujo celular.

El rol de S. pneumoniae resistente a PNC en las eventuales fallas de tratamiento en infecciones respiratorias ha sido la principal causa esgrimida, con o sin sustento microbiológico, para indicar otros antimicrobianos, tales como cefalosporinas de segunda y tercera generación de uso oral, de amoxicilina (AMX) + inhibidores de $\beta$ lactamasas y de macrólidos, considerando, como es tradicional, que la resistencia a PNC implica resistencia a otros antimicrobianos del mismo grupo, incluida la AMX.

Pese a esta observación teórica, en la práctica clínica los pacientes tratados con AMX a dosis de 80 a 100 $\mathrm{mg} / \mathrm{kg} / \mathrm{día}$ responden satisfactoriamente, con mínimos fracasos terapéuticos. Esta observación clínica indujo a nuestro grupo a comparar la CIM de $S$. pneumoniae a PNC y a AMX en 187 cepas aisladas desde el fluido de oído medio en niños con OMA. Pudimos corroborar la descrito por otros autores; que la susceptibilidad a PNC y AMX no son equivalentes. En efecto, en esta serie la susceptibilidad in vitro a PNC y AMX era de 60 y $94,7 \%$, la resistencia intermedia de 22 y $4,8 \%$, y la resistencia de alto nivel de 18 y $0,5 \%$ respectivamente (Tabla 2). Este hallazgo avala la recomendación de no extrapolar CIM de PNC a AMX, teniendo impacto clínico en el uso racional de antimicrobianos.

\section{Tratamiento}

Hoy en día, existen dos tendencias en el manejo de niños con OMA: Observación por 48 horas, e indicación de tratamiento antimicrobiano frente a la ausencia de mejoría clínica o, alternativamente, tratamiento antimicrobiano de inmediato, una vez hecho el diagnóstico. Las evidencias que avalan la primera conducta provienen de estudios de observación, de cohorte y randomizados (tratamiento versus placebo) en que la presencia de complicaciones, como meningitis y mastoiditis, fue igual en el grupo tratado con y sin antimicrobianos. Las limitaciones de estos estudios son la 
falta de inclusión de lactantes bajo seis meses de edad y de niños con enfermedad grave. La recomendación de consenso de la Cochrane Library al respecto, publicada el año 2004, plantea el tratamiento antimicrobiano de la OMA como opción definitiva, en niños bajo seis meses de edad. La posibilidad de observación, también es clara en niños sobre dos años de edad y deja un terreno incierto en los pacientes entre 6 y 24 meses, en que ambas posibilidades son recomendables de acuerdo con la certeza diagnóstica. En la opinión de esta autora, se debe hacer énfasis en el diagnóstico lo más certero posible de OMA en pediatría y frente al diagnóstico confirmado de una OMA, a cualquier edad, se debe presuponer una etiología bacteriana y tratar al paciente. Cualquiera de las dos conductas que un clínico tome en forma responsable implica, necesariamente, el control de ese niño para seguir el algoritmo de manejo recomendado (Figura 1).

\section{Esquemas terapéuticos}

Consideraciones generales. El mejor tratamiento antimicrobiano es aquel que está indicado de acuerdo con una base microbiológica, es eficiente para erradicar el microorganismo causante de la patología, tiene un espectro de acción restringido, no hace daño a la ecología microbiana, es bien tolerado, es de bajo costo y su uso no provoca una presión selectiva para favorecer la resistencia bacteriana.

La emergencia de resistencia a antimicrobianos es considerada un problema relevante en Chile y, al respecto, se deben adoptar estrategias que favorezcan el uso racional de éstos, tanto en la idea de contener el aumento de la resistencia, como en la de racionalizar los costos de salud para el Estado y para los individuos.

Relacionado con lo anterior, si se decide tratar un paciente con OMA, la indicación de manejo es AMX, $80 \mathrm{mg} / \mathrm{kg} /$ día, fraccionado cada $12 \mathrm{hrs}$. La indicación de fraccionar cada 12 horas se basa en el concepto de que los antimicrobianos $\beta$ lactámicos conservan su efecto bactericida en OMA siempre que, durante al menos $40 \%$ del tiempo que media entre dos dosis del mismo, su concentración en el oído medio supere la $\mathrm{CIM}_{90}$. El período de tratamiento con AMX se mantiene en 10 días en el lactante y se aceptan hoy tratamientos acortados (5-7 días de AMX) en niños sobre este límite de edad, sin OMA en el último mes, en ausencia de supuración.

Como alternativa de tratamiento, frente a una falla del mismo, definida como la ausencia de mejoría clínica de síntomas y signos (otalgia, fiebre, irritabilidad, otorrea o signos otoscópicos) luego de tres días de tratamiento o el reinicio de síntomas y signos dentro de los primeros siete días de tratamiento en un paciente que experimentó una mejoría clínica, se propone el uso de AMX + inhibidos de $\beta$ lactamasas o cefalosporinas de segunda generación; ambas indicaciones sólo en ambientes con alta prevalencia de $H$. influenzae productor $\beta$ lactamasa y M. catarrhalis; macrólidos, sabiendo que presentan $10 \%$ de resistencia de $S$. pneumoniae en niños con OMA en nuestro medio y pobre acción frente a $H$. influenzae; cloranfenicol, alternativa interesante frente al fracaso terapéutico de AMX, por su actividad frente a $S$. pneumoniae, $H$. influenzae y $S$. pyogenes; y ceftriaxona, como una medida de extrema excepción, en niños que no mejoran con cualquiera de las alternativas anteriores (Figura 1).

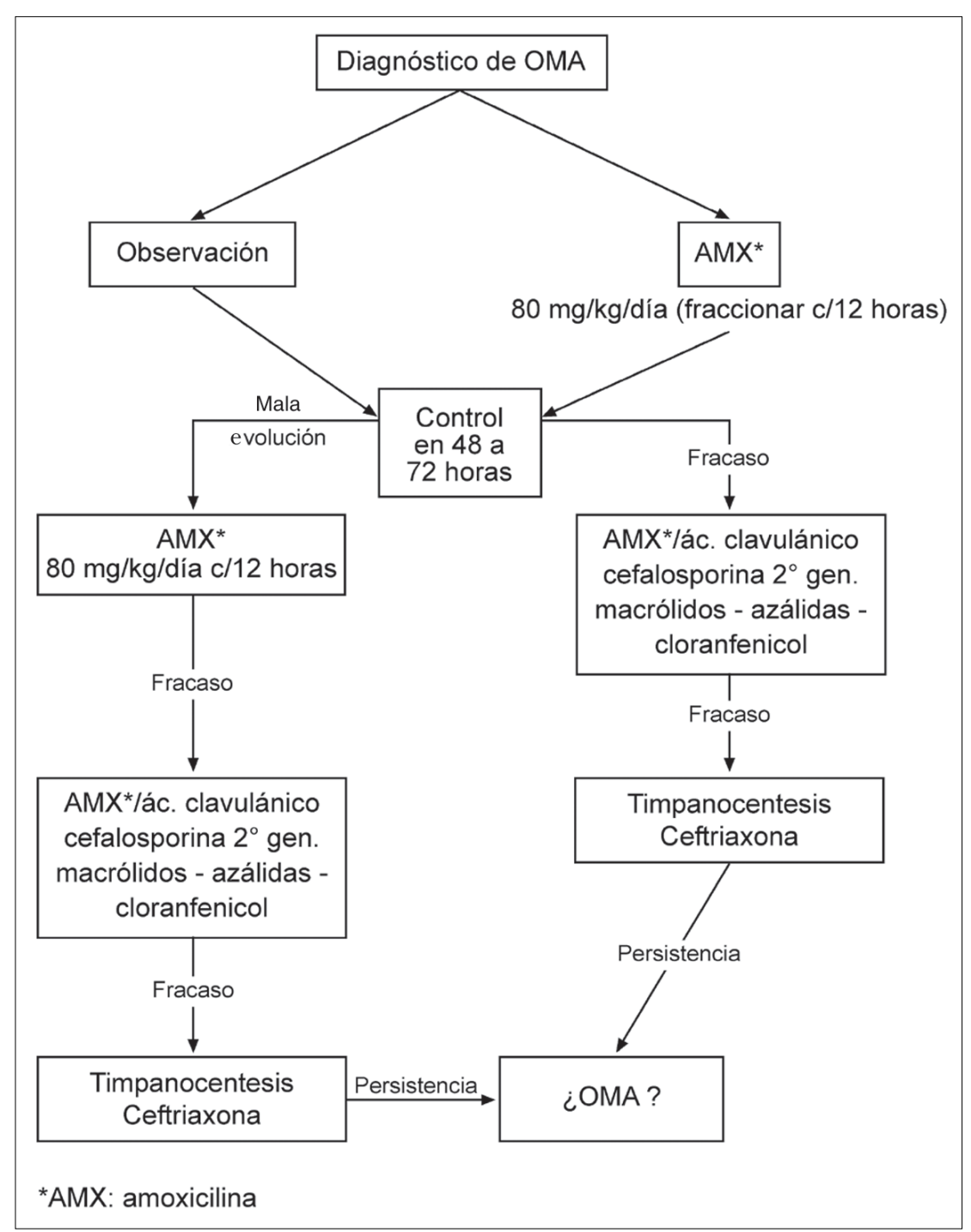

Figura 1. Algoritmo de manejo de niños con otitis media aguda. 


\section{Resumen}

Otitis media aguda (OMA) es una de las principales causas de consulta y de indicación de antimicrobianos en pediatría. El manejo racional de esta patología implica un diagnóstico acucioso y un conocimiento actualizado de las especies bacterianas involucradas en su etiología y de su susceptibilidad a antimicrobianos. El diagnóstico es clínico, a través de neumo-otoscopia. Los principales agentes involucrados en OMA en estudios hechos en Santiago de Chile son Streptococcus pneumoniae (40\%), Haemophilus influenzae no capsulado (29\%), Streptococcus pyogenes (7\%) y Moraxella catarrhalis (4\%). S. pneumoniae ha adquirido resistencia a penicilina en la última década, lo que se ha extrapolado a otros $\beta$ lactámicos, como amoxicilina, y ha sido el principal argumento para indicar otros antimicrobianos en el manejo de esta patología. Pese a esta observación, en la práctica clínica los pacientes tratados con amoxicilina a dosis de 80 a $100 \mathrm{mg} / \mathrm{kg} / \mathrm{día}$ responden satisfactoriamente, con mínimos fracasos terapéuticos. Estudios actuales, hechos en nuestro medio muestran que la susceptibilidad a penicilina y amoxicilina no son equivalentes, mostrando resistencia de alto nivel en 18 y $0,5 \%$ respectivamente. El tratamiento recomendado hoy, de acuerdo con datos nacionales, es amoxicilina, $80 \mathrm{mg} / \mathrm{kg} / \mathrm{dí}$, fraccionada cada $12 \mathrm{hrs}$, por 10 días en el lactante y por 5-7 días en niños $>$ de 2 años, sin antecedentes de OMA a repetición. Como alternativa de tratamiento, frente a una falla del mismo, se propone el uso de amoxicilina + inhibidores de $\beta$ lactamasas o cefalosporinas de segunda generación en ambientes con alta prevalencia de $H$. influenzae productores de $\beta$ lactamasas y $M$. catarrhalis. El tratamiento de un niño con OMA implica, necesariamente, control y seguimiento hasta su mejoría.

\section{Referencias}

1.- Rosenblüt A, Santolaya M E, González P, Corbalán V, Avendaño L, Martinez M A, Hormazabal J C. Bacterial and viral etiology of acute otitis media in Chilean children. Pediatr Infect Dis J 2001; 20: 501-7.

2.- Rosenblüt A, Santolaya M E, González P, Borel C, Cofré J. Penicillin resistance is not extrapolable to amoxicillin resistance in
Streptococcus pneumoniae isolated from middle ear fluid in children with acute otitis media. Annals of Otol, Rhinol and Laryngol 2006; 115: 186-90.

3.- American Academy of Pediatrics Subcommittee of Management of Acute Otitis Media. Diagnosis and management of acute otitis media. Pediatrics 2004; 113 : 1451-65.

4.- Teele D W, Klein J, Rosner B. Epidemiology of otitis media in the first seven years of life in children Greater Boston: a prospective, cohort study. J Infect Dis 1989; 160: $83-94$.

5.- Siegel R, Bien J. Acute otitis media in children. A continuing story. Pediatr Rev 2004; 25: 187-93.

6.- Dagan R, Johnson C, McLinn S. Bacteriologic and clinical efficacy of amoxicillin/clavulanate versus azithromycin in acute otitis media. Pediatr Infect Dis J 2000; 19: $95-104$ 